\title{
The relation between angioarchitectural factors of developmental venous anomaly and concomitant sporadic cavernous malformation
}

Tengfei Yu, Xing Liu, Xiangjiang Lin, Chuanfeng Bai, Jizong Zhao, Junting Zhang, Liwei Zhang, Zhen Wu, Shuo Wang, Yuanli Zhao and Guolu Meng ${ }^{*}$

\begin{abstract}
Background: Past studies found that cerebral developmental venous anomaly (DVA) is often concurrent with cavernous malformation (CM). But the reason of the concurrency remains unknown. The purpose of this study was to confirm whether angioarchitectural factors relate to the concurrence and which angioarchitectural factors can induce the concurrency.

Methods: DVA cases were selected from the records of the same 3.0 T MR. The DVA cases was divided into two group which are DVA group and DVA concurrent with CM group. 8 angioarchitectural factors of the DVAs were selected and measured. Statistical analysis was performed by the Pearson chi-square statistic,analysis of variance (ANOVA) and multi-factor logistic regression analysis.

Results: Five hundred three DVA lesions were found and 76 CM lesions coexisting with DVA. In the single factor analysis, all the 8 angioarchitectural factors of DVA were related to the concurrency. In the multivariate analysis, 6 angioarchitectural factors. Result of multi-factor logistic regression analysis is Logit( $(P)=-4.858-0.932$ (Location) + 1.616 (Direction) +1.757 (Torsion) +0.237 (Number) +2.119 (Stenosis rate of medullary vein)-0.015(Angle), goodness of fit is $90.1 \%$.
\end{abstract}

Conclusions: The angioarchitectural factors of DVA are related to the concurrency of DVA and CM. 6 angioarchitectural factors may induce the concurrency.

Keywords: Developmental venous anomaly (DVA), Cavernous malformation (CM), Angioarchitectural factors, Contrast-enhanced magnetic resonance imaging

\section{Background}

There are four major types of vascular malformations of the central nervous system: developmental venous anomaly (DVA), cavernous malformation (CM), arteriovenous malformation(AVM), and capillary telangiectasia [1]. Previous studies indicate that DVA and CM are the two most common central nervous system diseases among vascular malformations, and are frequently found coexisting.

\footnotetext{
* Correspondence: mengguolu@sina.com

Department of Neurosurgery, Beijing Tiantan Hospital,Capital Medical University, China National Clinical Research Center for Neurological Diseases, Center of Stroke, Beijing Institute for Brain Disorders, Beijing Key Laboratory of Translational Medicine for Cerebrovascular Disease, 6 Tiantan Xili, Chongwen District, Beijing 100050, People's Republic of China
}

DVA makes up most of all cerebral vascular malformations (CVM) and Our previous studies found that concurrent CM is more likely to form when a DVA has three or more medullary veins that are visible simultaneously in at least one MRI section [2-4].

Developmental venous anomaly (DVA) (also referred to as venous angiomas or venous malformations) is usually discovered incidentally on enhanced CT or brain magnetic resonance imaging (MRI) with a reported incidence of $0.05-2.56 \%$ in the general population $[5,6]$. DVAs are a congenital abnormality of venous drainage [7]. The lesion are composed of radially arranged venous complexes converging to a centrally located venous 
trunk, which drains the normal brain parenchyma [8]. Characteristically, DVAs have numerous dilated deep medullary veins presenting in "spoke wheel" or caput medusae configurations, which drain into a few dilated deep and/or superficial veins [9].

CMs are discrete well-circumscribed lesions formed by sinusoidal vascular spaces lined by a thin, single layer of endothelium of varying size and separated by a collagenous matrix devoid of elastin, smooth muscle, or other vascular wall elements $[4,10]$. They lack the microscopic features of arteries or veins.

$\mathrm{CM}$ is reported in the literature to have an association with DVA at a rate of $2-33 \%$ [7, 11-14]. Although there are no acknowledge theory to explain the anomaly high rate. Some studies show that the angioarchitectural factors of DVA are related to the concurrency of DVA and CM [2, 15]. High vein pressure and flow disturbance within the territory of DVA by the anatomical angioarchitectural factors may be key factors in leading to a cascade of events and subsequent development of a CM lesion [15]. But there are only a few correlative researches, which just studied a little part of angioarchitectural factors. In this study, analyze the factors (especially the angioarchitectural factors of DVA) associated with concurrency of these two diseases was aimed.

\section{Methods}

\section{Study population and data collection}

From January 1, 2007 to December 31, 2012, data were collected from the patients' MRI registration system and MRI reports form the same 3.0 T MRI unit at Beijing Tiantan Hospital. During the study period, the following MRI machines used were: GE Signa 3.0 T, superconducting magnetic resonance imager. The contrast agent was Gadopentetate dimeglumine (Gd-DTPA). At a rate of $1 \mathrm{ml} / \mathrm{sec}$ via an 18-gauge peripheral intravenous catheter, Gd-DTPA was bolus-injected by an MR power injector. The dose was $0.2 \mathrm{mmol} / \mathrm{kg}$. After $8 \mathrm{~s}$ of delay from the start of injection, the images were acquired.

For patients who underwent multiple MRI screenings over the study period, only the most recent screening results were included in this analysis. According to the standard procedures of Beijing Tiantan Hospital, all MRI images were analysed by two radiologists. The final diagnosis was approved by both radiologists. In rare cases when the radiologists' diagnosis was inconclusive, the researcher examined the original MRI images and assigned a classification to the case.

\section{Diagnostic criteria using MRI}

DVA diagnostic criteria included presence of lesions in the white matter, typical stellate or linear vascular lesions converging into a collecting vein and draining into the dural, sinus, or deep veins, and an umbrella or caput medusa-like appearance especially on an enhanced image [7].

CM diagnostic criteria included presence of lesions with reticulated mixed signal blood-containing locules with the classic heterogeneous "popcorn" appearance on both T1 and T2-weighted images, a rim of haemosiderin in the surrounding brain parenchyma; and minimal or no enhancement on the T1 image [7, 9]. Their appearance on a MRI will depend on the degree of the hemorrhage, with $\mathrm{T} 2$-weighted images being the most sensitive sequence. On imaging, the diagnosis of $\mathrm{CM}$ is one of exclusion; other causes of a single haemorrhagic lesion, such as arteriovenous malformation, bland intraparenchymal haemorrhage, haemorrhagic infection, and neoplasm must be excluded [16].

Hemorrhages were not classified as CMs if the hemorrhage lesions were only acute or subacute hematomas dominated by intracellular methaemoglobin, and therefore, appeared with a homogeneous signal on MRI images; and if there were only tiny, punctate foci of hypo intensity on both $\mathrm{T} 1$ and T2-weighted sequences, with no heterogeneous signal. On imaging, when CM diagnosis was made, other causes of a single hemorrhagic lesion, such as arteriovenous malformation, bland intraparenchymal hemorrhage, hemorrhagic infection, and neoplasm had to be excluded [17]. Figure 1 (This is a 18-year-old femal. It's the typical MRI images for DVA coexist with CM.)

\section{Image analysis}

All MR images should include: 1 transverse and reformatted sagittal, coronal enhanced T1-weighted images .2 transverse and reformatted sagittal T1-weighted images. 3 transverse T2-weighted images. They were analyzed by two researchers retrospectively, using the Picture Archiving and Communication Systems (PACS) without any prior information. The final result was the mean value of two researchers' measurement [17-20]. All the length, diameter, angle were measured by tools form the PACS.

Data of 8 angioarchitectural factors were collected. They are location of DVA(supratentorial or infratentorial), direction of the draining vein, torsion of draining vein, number of medullary veins, stenosis rate of draining vein, stenosis rate of medullary veins, length of draining vein, angle of the draining vein. Defination of the 8 angioarchitectural factors is as follow.

\section{Factor 1 location of DVA}

Two types of the location of the DVA lesion were defined, which were supratentorial or infratentorial.

Factor 2 Direction of the draining vein Fig. 2 (A is a 57-year-old DVA female patient. B is a 41-year-old DVA with concurrent $\mathrm{CM}$ male patient. $\mathrm{C}$ is the same patient 


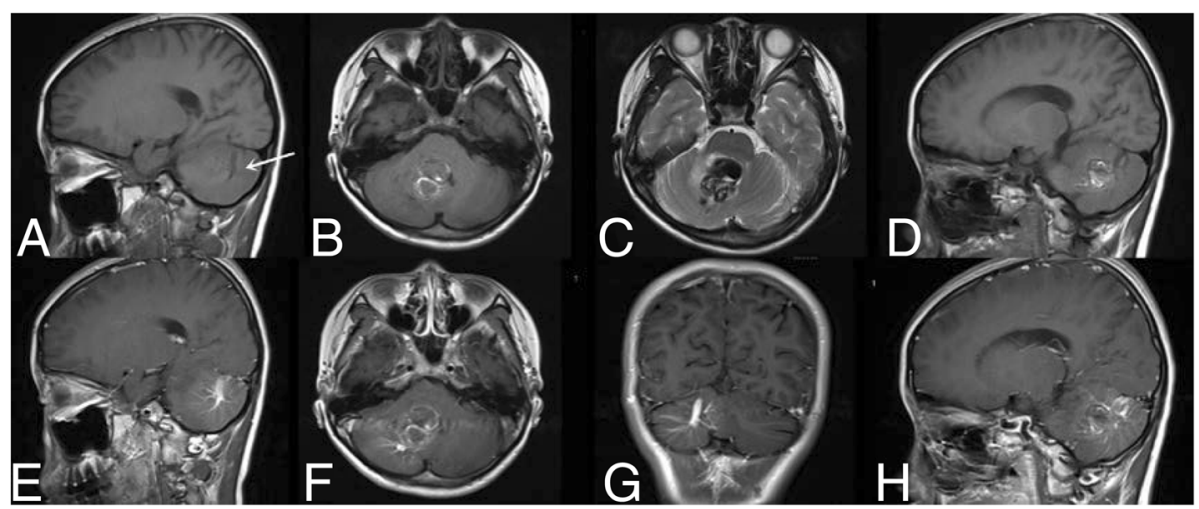

Fig. $\mathbf{1}$ This is a 18-year-old female. It's the typical MRI images for DVA coexist with CM. a b c $\mathbf{d}$ is the T1 weighted image, and $\mathbf{e} \mathbf{f} \mathbf{g} \mathbf{h}$ is the enhanced image. The arrow refers to DVA

with Fig. 1. D is a 35 years-old DVA male patient. The red arrows in A,B,C,D is the tool of PACS and it show the direction of draining. A is supratentorial DVA and superficial draining, B is supratentorial DVA and deep draining, $C$ is infratentorial DVA and superficial draining, D is infratentorial DVA and deep draining.)

The terminal or draining vein to which the caput medusae joins was classified as either a deep or superficial draining vein.

DVAs were defined as vascular lesions with multiple enlarged medullary veins converging on a single (sometimes multiple) dilated draining vein. All the DVA lesions have just one single draining vein in this research. So all six MRI sequences were analyzed and classified them. In the supratentorial compartment, superficial draining veins were identified as those that joined either a cortical vein or the sagittal sinus. Deep draining veins were identified as those that joined the subependymal veins of the lateral ventricles and ultimately the vein of Galen.

In the infratentorial compartment, superficial draining veins were identified as those that joined the cerebellar hemispheric veins, superior and inferior vermian veins,transverse or sigmoid sinus, and torcula. Deep draining veins were those that joined the subependymal veins of the fourth ventricle and thus either the anterior or lateral transpontine veins, or laterally and inferiorly to the veins of the lateral recess of the fourth ventricle, or superiorly to the precentral cerebellar vein $[3,12]$.

Factor 3 Torsion of draining vein Fig. 3 (This is a 41year-old patient who had DVA coexisting with CM. In this enhanced T1 image, two angles were found. Using the PACS tool(the red angles and yellow numbers), their angle were measured which are $70^{\circ}$ and $75^{\circ}$. This DVA lesion had two angle less than $120^{\circ}$ in the same section so it was defined as having torsion of the draining vein. Also the angle of draining vein was measured as $70^{\circ}$.)

Clinical routine MRI sequences was used for measuring the torsion. Because the are much more easily available than the $3 \mathrm{D}$ vision of draining vein. So the torsion factor was defined as this.

If the draining vein of DVAs has at least two angles less than $120^{\circ}$ in the same one section of the enhanced T1 MRI sequences, it was considered to have draining venous tortuosity.

Factor 4 Number of medullary veins Fig. 4 (This is the same patient with Fig. 1. The red arrows show the medullary veins of the DVAs. A(9)、B(4)、C(4) had the most medullary veins in their sequences respectively. A had the most ones. So the number of medullary veins of this patient is 9).

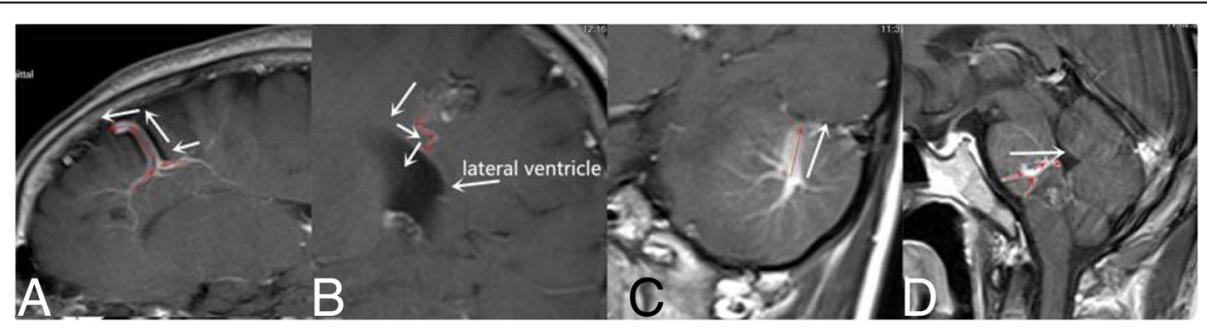

Fig. $\mathbf{2}$ a is a 57-year-old DVA female patient. $\mathbf{b}$ is a 41-year-old DVA with concurrent CM male patient. $\mathbf{c}$ is the same patient with Fig. 1. $\mathbf{d}$ is a 35 years-old DVA male patient. The red arrows in $\mathbf{a}, \mathbf{b}, \mathbf{c}, \mathbf{d}$ is the tool of PACS and it show the direction of draining. $\mathbf{a}$ is supratentorial DVA and superficial draining, $\mathbf{b}$ is supratentorial DVA and deep draining, $\mathbf{c}$ is infratentorial DVA and superficial draining, $\mathbf{d}$ is infratentorial DVA and deep draining 
A typical DVA lesion contains multiple enlarged medullary veins and a single (sometimes multiple) dilated draining vein. So number of the medullary veins of DVAs was defined as one of the 8 angioarchitectural factors. From different sections of enhanced T1 images, the DVA has different numbers of medullary veins. The maximum value of the numbers found from all the enhanced T1 images was defined as the number of the medullary veins of the DVAs.

Factor 5 Stenosis rate of draining vein Fig. 5 (A is the same patient with Fig. 1. B is a 35-year-old patient who had DVA coexisting with CM. Both of them are enhanced $\mathrm{T} 1$ images. In $\mathrm{B}$ the wildest diameter of medullary veins is $1.77 \mathrm{~mm}$, the wildest diameter of draining vein is $3.10 \mathrm{~mm}$, the narrowest diameter of draining vein is $1.29 \mathrm{~mm}$. So in B the stenosis rate of draining vein is $(1-1.29 / 3.10) \times 100 \%=58.39 \%$, the stenosis rate of medullary vein is $(1-1.77 / 3.10) \times 100 \%=42.90 \%$. In A, the same way was used).

In a DVA lesion, multiple enlarged medullary veins converging on a single dilated draining vein, which joins a sinus. According to the literature we put forward the hypothesis that stenosis rate of the draining vein can lead to the raise of venous pressure of DVA [11].

Using the PACS the widest and narrowest diameter of draining vein of all sections of enhanced T1 images were measured. The two diameters can be in the same section or different sections. Stenosis rate of the

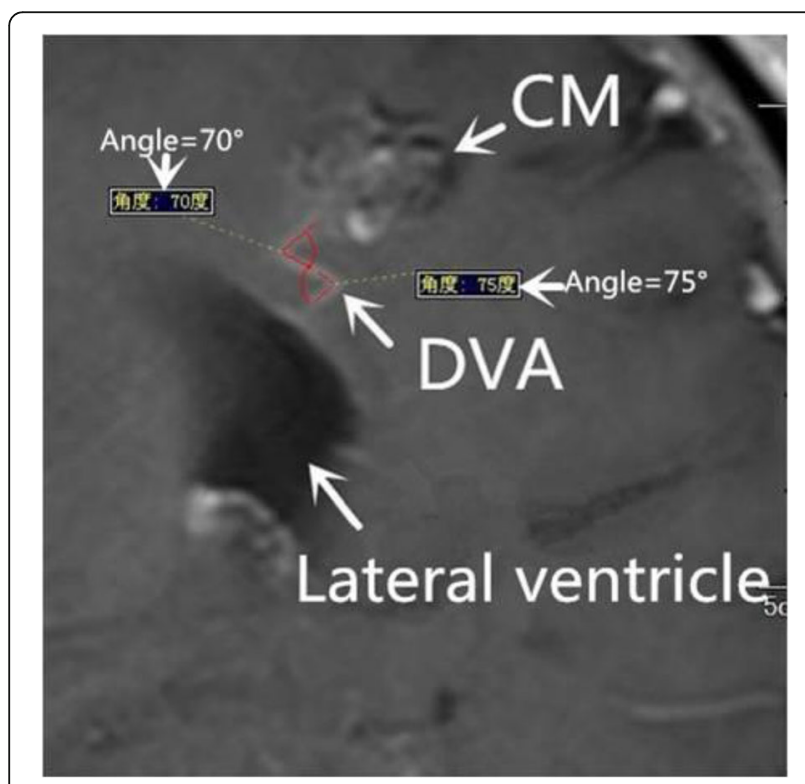

Fig. 3 This is a 41-year-old patient who had DVA coexisting with $\mathrm{CM}$. In this enhanced $\mathrm{T} 1$ image, two angles were found. Using the PACS tool(the red angles and yellow numbers), their angle were measured which are $70^{\circ}$ and $75^{\circ}$. This DVA lesion had two angle less than $120^{\circ}$ in the same section so it was defined as having torsion of the draining vein. Also the angle of draining vein was measured as $70^{\circ}$ draining vein $=(1-$ narrowest diameter $/$ widest diameter) $\times 100 \%$.

\section{Factor 6 Stenosis rate of medullary vein Fig. 5}

Similar to the Factor 5, the widest diameter of draining vein and medullary veins of all sections of enhanced $\mathrm{T} 1$ images were measured. The two diameters can be in the same section or different sections. Stenosis rate of the medullary veins $=(1-$ widest diameter of medullary veins/ widest diameter of draining vein) $\times 100 \%$.

\section{Factor 7 length of draining vein}

The caput of DVA is the part where medullary veins join the draining vein. The length of the caput to the end of draining vein is recorded in every section of enhanced T1 images. For the same reason of Factor 3 the maximum value was defined as the length of draining vein. The length of draining vein may have influence on the venous pressure $[2,3,15]$. If the draining vein was not straight, the length was measured part by part, using the PACS tools.

\section{Factor 8 angle of the draining vein Fig. 3}

Every angle of draining vein in all sections of enhanced $\mathrm{T} 1$ images were measured and define the minimum value as the angle of the draining vein.

\section{Statistical analyses}

For single-factor analysis, statistical analysis was performed using the Pearson chi-square statistic for binary variables which are Factor 1,2,3 and analysis of variance (ANOVA) for continuous variable which are Factor 4, 5, $6,7,8$.

Multi-factor logistic regression analysis were performed for predictors associated with the concurrency. In the Multi-factor logistic regression analysis, method was took as Forward:Conditional, Entry: $p<0.05$, Removal: $p>0.10 .8$ factors and independent variables included age, gender were analysised. For the age variable, age groups were formed by 20 year intervals $(\leq 20$, $20 \sim 40,40 \sim 60, \geq 60$ ). All confidence intervals reported were $95 \%$, and all p-values were two-sided. P-values less than 0.05 were considered statistically significant. All statistical analyses were performed using SPSS software (Version 17.0). The dummy variables were Gender, Age, Location of DVA, Direction of draining vein, Torsion of draining vein.

\section{Results}

Five hundred three DVA lesions met DVA radiographic criteria. 76 of 503 DVAs had coexisting $\mathrm{CM}$ and the other 427 DVAs lesion didn't have coexisting CM. In the single factor analysis, all the 8 angioarchitectural factors of DVA were found related to the concurrency $(p<0.05)$ 


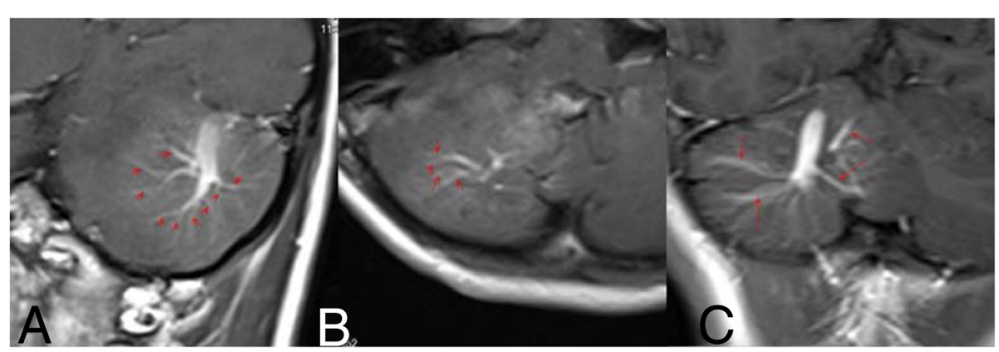

Fig. 4 This is the same patient with Fig. 1. The red arrows show the medullary veins of the DVAs. a (9), b (4), c (4) had the most medullary veins in their sequences respectively. $\mathbf{a}$ had the most ones. So the number of medullary veins of this patient is 9

and age $(p=0.800)$ or gender $(p=0.627)$ had no relation with it. Compared with the DVA group,DVA concurrent with CM group were more likely to locate in infratentorial area,vein drain into the deep area, have torsion of draining vein and they tend to have more medullary veins, higher stenosis rate of draining vein and medullary veins, longer draining vein and smaller angle of draining vein Table 1.

In the multivariate analysis, 6 angioarchitectural factors including DVA location (supratentorial or infratentorial), the direction of the draining vein, whether there was torsion of the draining vein,number of the medullary veins, stenosis rate of the medullary veins, angle of the draining vein were associated with concurrency of DVA and CM.

$\operatorname{Logit}(\mathrm{P})=-4.858-0.932$ (Location) +1.616 (Direction) +1.757 (Torsion) + 0.237 (Number) + 2.119 (Stenosis rate of medullary vein)-0.015 (Angle), goodness of fit is $90.1 \%$. Logit $(\mathrm{P})$ is the result of Multi-factor logistic regression analysis and it implies the odds or risk of a DVA lesion being concurrent with $\mathrm{CM}$. With the Logit(P) angioarchitectural factors can be transformed into numbers, which are more easily to tell a high risk DVA Table 2.
The angioarchitectural factors of DVA are related to the concurrency of DVA and CM. Especially, if the DVA follow the six angioarchitectural factors: locate in the infratentorial area, direction of the draining vein is deep, have torsion of draining vein, number of the medullary veins of DVAs $\geq 5$, stenosis rate of the medullary veins $\geq$ $54.68 \%$, angle of the draining vein $\leq 106.50^{\circ}$, it tend to have concurrent $\mathrm{CM}$.

\section{Discussion}

Characteristically, DVAs have numerous dilated deep medullary veins presenting in "spoke wheel" or caput medusae configurations, which drain into a few dilated deep and/or superficial veins [21-23] MRI is an optimal imaging tool for detecting CM and DVA [15, 24]. So in this study the two lesion were connect with clinical routine enhanced MRI data, which is easily available in the hospital instead of some other MRI sequences.

In a significant percentage of DVA cases, however, coexisting vascular malformations are found. The most common vascular anomaly associated with a DVA is $\mathrm{CM}$. The association between venous malformation and cavernous angioma has been recognized by many

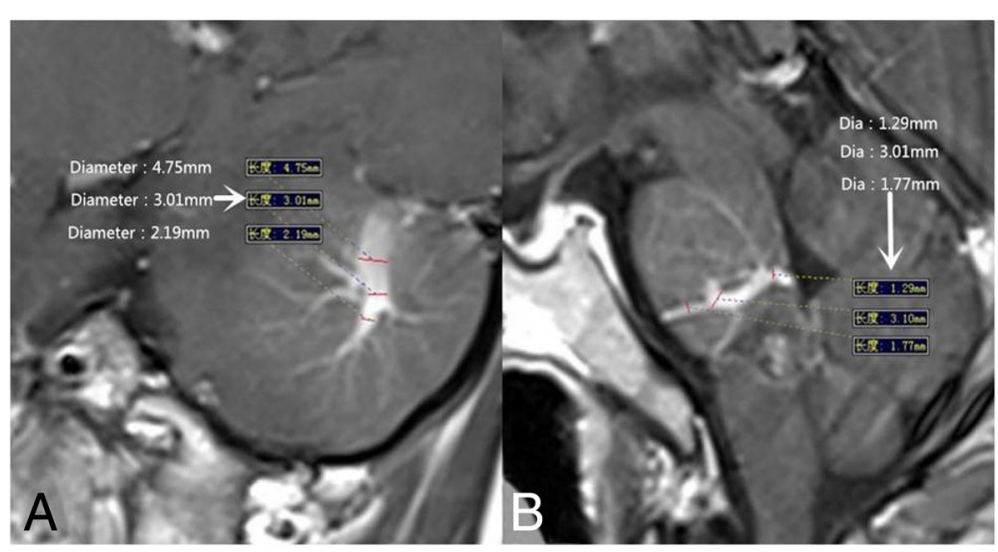

Fig. $\mathbf{5}$ a is the same patient with Fig. 1. $\mathbf{b}$ is a 35-year-old patient who had DVA coexisting with CM. Both of them are enhanced T1 images. In $\mathbf{b}$ the wildest diameter of medullary veins is $1.77 \mathrm{~mm}$, the wildest diameter of draining vein is $3.10 \mathrm{~mm}$, the narrowest diameter of draining vein is $1.29 \mathrm{~mm}$. So in $\mathbf{b}$ the stenosis rate of draining vein is $(1-1.29 / 3.10) \times 100 \%=58.39 \%$, the stenosis rate of medullary vein is $(1-1.77 / 3.10) \times$ $100 \%=42.90 \%$. In A, the same way was used 
Table 1 The result of single-factor analysis

\begin{tabular}{|c|c|c|c|c|}
\hline & & DVA with CM & DVA & $P$ \\
\hline \multirow[t]{2}{*}{ Gender } & Male & 37 (48.7 \%) & $195(45.7 \%)$ & 0.627 \\
\hline & Famle & 39 (51.3\%) & $232(54.3 \%)$ & \\
\hline \multirow[t]{4}{*}{ Age } & $<20$ & $8(10.5 \%)$ & $50(11.7 \%)$ & 0.800 \\
\hline & $20 \sim 40$ & $29(38.2 \%)$ & $180(42.2 \%)$ & \\
\hline & $40 \sim 60$ & $34(44.7 \%)$ & $165(38.6 \%)$ & \\
\hline & $\geq 60$ & $5(6.6 \%)$ & $32(7.5 \%)$ & \\
\hline \multirow[t]{2}{*}{ Location of DVA } & supratentorial & $35(46.1 \%)$ & $327(76.6 \%)$ & $<0.001$ \\
\hline & infratentorial & 41 (53.9\%) & $100(23.4 \%)$ & \\
\hline \multirow[t]{2}{*}{ Direction of draining vein } & Deep & $57(79.2 \%)$ & $181(42.4 \%)$ & $<0.001$ \\
\hline & Superficial & $19(20.8 \%)$ & $246(57.6 \%)$ & \\
\hline \multirow[t]{2}{*}{ Torsion of draining vein } & Positive & $50(65.8 \%)$ & $42(9.8 \%)$ & $<0.001$ \\
\hline & Negative & $26(34.2 \%)$ & $385(91.2 \%)$ & \\
\hline Number of medullary veins & & $5.91 \pm 0.33$ & $3.52 \pm 0.08$ & $<0.001$ \\
\hline Stenosis rate of draining vein & & $53.66 \pm 1.87 \%$ & $47.79 \pm 0.68 \%$ & $<0.001$ \\
\hline Stenosis rate of medullary veins & & $54.68 \pm 1.85 \%$ & $43.65 \pm 0.87 \%$ & $<0.001$ \\
\hline Length of draining vein(mm) & & $21.45 \pm 0.95$ & $16.36 \pm 0.40$ & $<0.001$ \\
\hline Angle of draining vein $\left(^{\circ}\right)$ & & $106.50 \pm 3.84$ & $136.62 \pm 3.84$ & $<0.001$ \\
\hline
\end{tabular}

authors. In our previous study of 1839 patients, the rate is $11.15 \%$ and 205 patients of the total 165,230 (1.24\%o) was found had DVA with concurrent CM [2]. Reports suggested that DVA in the posterior fossa are more likely to hemorrhage than are their supratentorial counterparts [25]. Concurrent CM is more likely when a DVA is infratentorial. Contrast-enhanced MR images have clearly shown that cavernous angiomas frequently appear to arise at the distal radicles of venous malformations $[2,11,14,26]$.

Reasons for the concurrency may be related with the vein pressure of DVA. Cortes [4] agree with the theory that high venous pressure of DVA induce the form of CM. There is increased systemic or local venous pressure in the DVA [27]. Increased venous pressure may lead to recurrent petechial congestional hemorrhage [28, 29], or may produce ischemia which stimulates the growth of new vessels [30]. There is high venous pressure in the DVAs and high venous pressure and may be the cause of hemorrhage from a DVA with subsequent formation of a CM. Progressive thickening of the walls of the DVA and their morphological organization into a venous convergence zone are thought to contribute to the development of venous hypertension in DVA. These new vessels are fragile and susceptible to bleeding, and repeated hemorrhaging may subsequently form a CM [15, 28, 31]. Maeder $\mathrm{P}$ et al. [27] found a high percentage of venous stenosis of the collector vein in the patients who have CM in association with DVA. San MRD et al. [12] reported that outflow obstruction by stenosis of collecting vein was demonstrated in $13.1 \%$ of the DVAs. Hong YJ et al [19]. found that anatomical angioarchitectural factors might be the key factors in causing concurrent sporadic CM within the territory of DVA by causing disturbance of blood flow. These

Table 2 The result of multi-factor logistic regression analysis

\begin{tabular}{|c|c|c|c|c|c|c|c|}
\hline & \multirow[t]{2}{*}{ B } & \multirow[t]{2}{*}{ S.E. } & \multirow[t]{2}{*}{ Wald } & \multirow[t]{2}{*}{$P$} & \multirow[t]{2}{*}{ OR } & \multicolumn{2}{|c|}{$95.0 \%$ C.I.for OR } \\
\hline & & & & & & Lower & Upper \\
\hline Location of DVA & -0.932 & 0.335 & 7.723 & 0.005 & 0.394 & 0.204 & 0.760 \\
\hline Number of medullary veins & 0.237 & 0.078 & 9.165 & 0.002 & 1.267 & 1.087 & 1.477 \\
\hline Stenosis rate of medullary veins & 2.119 & 1.076 & 3.876 & 0.049 & 8.321 & 1.009 & 68.591 \\
\hline Angle of draining vein & -0.015 & 0.006 & 5.310 & 0.021 & 0.985 & 0.973 & 0.998 \\
\hline Direction of draining vein & 1.616 & 0.362 & 19.984 & 0.000 & 5.034 & 2.478 & 10.224 \\
\hline Torsion of draining vein & 1.757 & 0.412 & 18.215 & 0.000 & 5.793 & 2.586 & 12.981 \\
\hline Constant & -4.858 & 1.125 & 18.643 & 0.000 & 0.008 & & \\
\hline
\end{tabular}


factors include angulation of the vein, narrowing of distal draining vein, severe medullary venous tortuosity. Increased systemic venous pressure or increased local venous pressure secondary to stenosis of the draining transparenchymal vein or other venous obstruction could lead to haemorragic or ischaemic complications. Also lead to recurrent petechial hemorrhage characteristic of cavernous angiomas. Thrombosis of the collecting veins and raised pressure in the territory drained by the DVA are the causes of congestional haemorrhage. This, in turn, further provides angiogenic factor and stimulates growth of new vessels. Neovasculature lacks vasoregulatory capacity and is fragile enough to make it susceptible to bleed. This leads to repeated hemorrhage and hence formation of abnormal vessels and eventually a CM would be formed. Elevated venous pressure within the territory of a venous malformation may provoke angiogenic factors that may be responsible for the recruitment of new vessels. It is difficult to measure the pressure of DVA, but the pressure has been recorded in CMs during surgery and found to be substantially higher than cortical venous pressure.

So factors that increase the venous pressure can induce the form of CM. Dillon et al [32] reported that the development of cryptic vascular malformation was related to elevated venous pressure, occurring within a venous malformation, a telangiectasis, or a minute vascular malformation consequent to venous outflow obstruction. They found capillary telangiectasias and transitional lesions at the periphery of cavernous malformations in autopsy series and elevated venous pressure in DVAs leads to ectasia in an acquired telangiectasia that evolves toward a cavernous malformation. With this theory, we believe that higher risk of concurrency is related to higher risk of hemorrhage of CM.

Other reason for the concurrency may be: DVAs undergo changes common to the ageing venous system that can lead to ischaemic phenomena and secondary haemorrhage may then occur [28]. It is likely that cavernous malformations, as identified on MR imaging, are an end result of several possible initiating causes of vascular change: DVA, perhaps with stenosis in 1 branch; radiation; and genetic factors such as the KRIT1 and other known mutations [10].

DVA location (supratentorial or infratentorial), the direction of vein draining, torsion of draining vein, number of medullary veins, rate of stenosis of medullary veins, xangle of draining vein are the six angioarchitectural factors were found. Some studies show that the infratentorial DVAs may have higher venous pressure, because they are deep and have narrow space. When the draining vein have severe venous tortuosity, the venous pressure may be higher than other DVAs.
With more medullary veins, DVA may have more venous blood in the lesion and that will lead to higher venous pressure. They can raise the venous pressure and thus raise the mobidity of DVA concurrent with sporadic CM. So angioarchitectural factors which cause disturbance of blood flow might be the key factors in causing concurrent sporadic $\mathrm{CM}$ within the territory of DVA [18, 33-35].

Here are the deficiencies of this research. The study focus on the MRI data of DVA and CM and didn't contain the patients' clinical symptom and sign or conditions of prognosis. Clinical correlations and detailed follow-up are limited and are beyond the scope of this imaging-based study. Because only the routine MRI sequences were used, the result data may have deviation with the true data of every angioarchitectural factors.

\section{Conclusions}

This study shows that if the DVA follow the six angioarchitectural factors :locate in the infratentorial area, direction of the draining vein is deep, have torsion of draining vein, number of the medullary veins of DVAs $\geq 5$, stenosis rate of the medullary veins $\geq 54.68 \%$, angle of the draining vein $\leq 106.50^{\circ}$,it tend to have concurrent sporadic $\mathrm{CM}$. The concurrent morbidity can be predicted with the following equation. $\operatorname{Logit}(\mathrm{P})=-4.858-0.932($ Location $)+1.616($ Direction $)+1.757$ (Torsion) +0.237 (Number) +2.119 (Stenosis rate of medullary vein)-0.015(Angle), goodness of fit is $90.1 \%$.

\section{Abbreviations}

ANOVA: Analysis of variance; AVM: Arteriovenous malformation; CM: Cavernous malformation; CVM: Cerebral vascular malformations; DVA: Developmental venous anomaly; Gd-DTPA: Gadopentetate dimeglumine; MRI: Magnetic resonance imaging; PACS: Picture Archiving and Communication Systems

\section{Acknowledgements \\ The study received financial support from the Beijing Nova program of science and technology, National Key Technology Research and Development Program of the Ministry of Science and Technology of China (2013BAI09B03) and Center of Stroke, Beijing Institute for Brain Disorders (BIBD-PXM2013_014226_07_000084). The funding organizations had no role in development of the study or the drafting of the manuscript for publication.}

\section{Availability of data and materials}

Data of the research will not be shared,because Beijing Tiantan Hospital forbid that.

\section{Authors' contributions}

Dr. TFY was responsible for the study design, statistical analysis of the data, and the drafting of this article. Dr. XL and Dr. XJL were responsible for collection of the clinical and imaging data, and Dr. CFB was responsible for the literature review. Dr. JTZ,Dr. JZZ, Dr. LWZ, Dr. ZW, Dr. SW and Dr. YLZ were responsible for the study design, critical revision of the draft, and the final approval of the version to be published. Dr. GLM was responsible for data interpretation, and review and revision of the draft. All authors read and approved the final manuscript. 


\section{Authors' information}

The First author now works at Department of Neurosurgery, Peking University International Hospital, 6 Shengmingkexueyuan Road, Changping District, Beijing 102206, People's Republic of China.

\section{Competing interests}

The authors declare that they have no competing interests.

\section{Consent for publication}

Not applicable.

\section{Ethics approval and consent to participate}

The study was approved by the Committee on Human Research at Beijing Tiantan Hospital.

Received: 19 March 2016 Accepted: 29 August 2016

\section{Published online: 22 September 2016}

\section{References}

1. Russell DS, Rubenstein L. Pathology of Tumors of the Nervous System. London: Edward Arnold; 1977. pp. 78-79.

2. Meng G, Bai C, Yu T, et al. The association between cerebral developmental venous anomaly and concomitant cavernous malformation: an observational study using magnetic resonance imaging. BMC Neurol. 2014;14:50.

3. Koc K, Anik I, Akansel Q, Anik Y, Ceylan S. Massive intracerebral haemorrage due to developmental venous anomaly. Br J Neurosurg. 2007;21(4):403-5.

4. Cortes VJJ, Concepcion AL, Ballenilla MF, Gallego LII, Gonzalez-Spinola SGJ. Cerebral cavernous malformations: spectrum of neuroradiological findings. Radiologia (Panama). 2012;54(5):401-9.

5. Hon JM, Bhattacharya JJ, Counsell CE, et al. The presentation and clinical course of intracranial developmental venous anomalies in adults: a systematic review and prospective, population-based study. Stroke. 2009:40(6):1980-5.

6. Ostertun B, Solymosi L. Magnetic resonance angiography of cerebral developmental venous anomalies: its role in differential diagnosis. Neuroradiology. 1993;35(2):97-104.

7. Ruíz DS, Yilmaz H, Gailloud P. Cerebral developmental venous anomalies: current concepts. Annals of Neurology. 2009;66(3):271-83.

8. Fushimi Y, Miki Y, Togashi K, Kikuta K, Hashimoto N, Fukuyama H. A developmental venous anomaly presenting atypical findings on susceptibility-weighted imaging. AJNR Am J Neuroradiol. 2008;29(7):E56.

9. Vattoth S, Purkayastha S, Jayadevan ER, Gupta AK. Bilateral cerebral venous angioma associated with varices: a case report and review of the literature. AJNR Am J Neuroradiol. 2005:26(9):2320-2.

10. Petersen TA, Morrison LA, Schrader RM, Hart BL. Familial versus sporadic cavernous malformations: differences in developmental venous anomaly association and lesion phenotype. AJNR Am J Neuroradiol. 2010:31(2):377-82.

11. Wurm G, Schnizer M, Nussbaumer K, Wies W, Holl K. Recurrent cryptic vascular malformation associated with a developmental venous anomaly. $\mathrm{Br}$ J Neurosurg. 2003;17(2):188-95.

12. San MRD, Delavelle J, Yilmaz $H$, et al. Parenchymal abnormalities associated with developmental venous anomalies. Neuroradiology. 2007:49(12):987-95.

13. Perrini $P$, Lanzino $G$. The association of venous developmental anomalies and cavernous malformations: pathophysiological, diagnostic, and surgical considerations. Neurosurg Focus. 2006;21(1):e5

14. Rammos SK, Maina R, Lanzino G. Developmental venous anomalies: current concepts and implications for management. Neurosurgery. 2009;65(1):20-9; discussion 29-30.

15. Patel VJ, Lall RR, Desai S, Mohanty A. Spontaneous Thrombosis and Subsequent Recanalization of a Developmental Venous Anomaly. Cureus. 2015;7(9):e334

16. Hegde AN, Mohan S, Lim CC. CNS cavernous haemangioma: "popcorn" in the brain and spinal cord. Clin Radiol. 2012:67(4):380-8.

17. Abe T, Singer RJ, Marks MP, Norbash AM, Crowley RS, Steinberg GK. Coexistence of occult vascular malformations and developmental venous anomalies in the central nervous system: MR evaluation. AJNR Am J Neuroradiol. 1998;19(1):51-7.

18. Lee C, Pennington MA, Kenney 3rd CM. MR evaluation of developmental venous anomalies: medullary venous anatomy of venous angiomas. AJNR Am J Neuroradiol. 1996;17(1):61-70.
19. Hong YJ, Chung TS, Suh SH, et al. The angioarchitectural factors of the cerebral developmental venous anomaly; can they be the causes of concurrent sporadic cavernous malformation. Neuroradiology. 2010;52(10):883-91.

20. Sughrue ME, Connolly Jr ES. Possible mechanistic overlap between cavernous malformations and cerebral developmental venous anomalies. Stroke. 2005:36(11):2339.

21. Lasjaunias P, Burrows P, Planet C. Developmental venous anomalies (DVA): the so-called venous angioma. Neurosurg Rev. 1986;9(3):233-42.

22. Topper R, Jurgens E, Reul J, Thron A. Clinical significance of intracranial developmental venous anomalies. J Neurol Neurosurg Psychiatry. 1999;67(2):234-8.

23. Sirin S, Kahraman S, Gocmen S, Erdogan E. A rare combination of a developmental venous anomaly with a varix. Case report. J Neurosurg Pediatr. 2008:1(2):156-9.

24. Porter RW, Detwiler PW, Spetzler RF, et al. Cavernous malformations of the brainstem: experience with 100 patients. J Neurosurg. 1999;90(1):50-8.

25. Truwit CL. Venous angioma of the brain: history, significance, and imaging findings. AJR Am J Roentgenol. 1992:159(6):1299-307.

26. Wilms G, Bleus $E$, Demaerel $P$, et al. Simultaneous occurrence of developmental venous anomalies and cavernous angiomas. AJNR Am J Neuroradiol. 1994:15(7):1247-54. discussion 1255-7.

27. Maeder P, Gudinchet F, Meuli R, de Tribolet N. Development of a cavernous malformation of the brain. AJNR Am J Neuroradiol. 1998:19(6):1141-3.

28. Hussain JZ, Ray A, Hughes DG, Leggate JR. Complex developmental venous anomaly of the brain. Acta Neurochir (Wien). 2002:144(5):501-4.

29. Wilson CB. Cryptic vascular malformations. Clin Neurosurg. 1992;38:49-84.

30. Rothbart D, Awad IA, Lee J, Kim J, Harbaugh R, Criscuolo GR. Expression of angiogenic factors and structural proteins in central nervous system vascular malformations. Neurosurgery. 1996;38(5):915-24. discussion 924-5.

31. Leblanc GG, Golanov E, Awad IA, Young WL. Biology of vascular malformations of the brain. Stroke. 2009;40(12):e694-702.

32. Dillon WP. Cryptic vascular malformations: controversies in terminology, diagnosis, pathophysiology, and treatment. AJNR Am J Neuroradiol. 1997:18(10):1839-46

33. Rammos SK, Maina R, Lanzino G. Developmental venous anomalies: current concepts and implications for management. Neurosurgery. 2009:65(1):20-9. discussion 29-30.

34. Abdulrauf SI, Kaynar MY, Awad IA. A comparison of the clinical profile of cavernous malformations with and without associated venous malformations. Neurosurgery. 1999:44(1):41-6. discussion 46-7.

35. Santucci GM, Leach JL, Ying J, Leach SD, Tomsick TA. Brain parenchymal signal abnormalities associated with developmental venous anomalies: detailed MR imaging assessment. AJNR Am J Neuroradiol. 2008;29(7):1317-23.

\section{Submit your next manuscript to BioMed Central and we will help you at every step:}

- We accept pre-submission inquiries

- Our selector tool helps you to find the most relevant journal

- We provide round the clock customer support

- Convenient online submission

- Thorough peer review

- Inclusion in PubMed and all major indexing services

- Maximum visibility for your research

Submit your manuscript at www.biomedcentral.com/submit
Biomed Central 